Pacific Journal of Mathematics

ON THE EXTENSION OF CONTINUOUS AND ALMOST

PAU Mites 


\title{
ON THE EXTENSION OF CONTINUOUS AND ALMOST PERIODIC FUNCTIONS
}

\author{
Paul Milnes
}

The most important results of this paper are two not very closely related theorems concerning the extension of functions. For the first theorem, let $A$ be a subspace of a topological space $B$ and let $X$ and $Y$ be subsets of $C(A)$ and $C(B)$. respectively. The theorem then asserts that, if every member of $X$ extends to a member of $Y$, then every member of the $C^{*}$-subalgebra of $C(A)$ generated by $X$ extends without increase in norm to a member of the $C^{*}$-subalgebra of $C(B)$ generated by $Y$. As an application of this theorem, new proofs of some results of $J$. F. Berglund on the extension of almost periodic functions are given.

The statement of the second theorem is: every weakly almost periodic function on an open subgroup $H$ of a locally compact group $G$ extends to a function weakly almost periodic on $G$. (K. deLeeuw and I. Glicksberg have proved this result with the additional assumption that $H$ is normal.)

1. Introduction. A consequence of Pontryagin's duality theorem is as follows:

(A) every continuous character on a closed subgroup $H$ of a locally compact abelian group $G$ admits an extension to a continuous character of $G$ [5; (24.12) Corollary, p. 380].

It is not at all clear why (A) should imply

(B) every function in $A P(H)$, the space of almost periodic functions on $H$ (which in this case is just the $C^{*}$-subalgebra of $C(H)$ generated by the continuous characters on $H$ ), admits an extension to a function in $A P(G)$.

That (B) does hold is a theorem of Berglund [1; Corollary 11]; the result (A) plays an essential role in his proof. That $(B)$ holds may also be deduced from (A) and the following general theorem which is the main result of $\$ 2$.

THEOREM 1. Let $A$ be a subspace of a topological space $B$ and let $X$ and $Y$ be subsets of $C(A)$ and $C(B)$ respectively. Then every member of the $C^{*}$-subalgebra of $C(A)$ generated by $X$ extends without increase in norm to a member of the $C^{*}$-subalgebra of $C(B)$ generated by $Y$ if

(*) every member of $X$ extends to a member of $Y$ 
(even. if there is a sequence $\left\{f_{n}\right\} \subset X, f_{n} \neq 0 \forall n$, such that $\inf \left\{\|F\| /\left\|f_{n}\right\| \mid F \in Y, F\right.$ is an extension of $\left.f_{n}\right\} \rightarrow \infty$ as $n \rightarrow \infty$ ).

The proof of the theorem uses some results from the theory of uniform spaces. These results can be found in Kelley's book [6] and elsewhere. Our notation and terminology for uniform spaces is also taken from [6].

In $\$ 3$, we concern ourselves with the statement

$(* *)$ every weakly almost periodic function on a subgroup $H$ of a locally compact group $G$ extends to a function weakly almost periodic on $G$.

After noting that $(* *)$ is not true in general, we mention three cases where (**) is known to be true and prove (**) is true if $G$ is locally compact and $H$ is compact or open (Proposition 1 and Theorem 2). The group of finite permutations of the natural numbers turns out to be an interesting example in this regard.

2. An extension theorem. Suppose $A$ is a topological space and $X$ is a subset of $C(A)$, the $C^{*}$-algebra of continuous bounded functions on $A$. Subsets of continuous function spaces dealt with in this paper are always assumed to contain a nonzero constant function. Given $X \subset C(A)$, we let $C^{*}(X)$ be the $C^{*}$-subalgebra of $C(A)$ generated by $X$ and denote by $\mathcal{U}(A, X)$, resp. $U\left(A, C^{*}(X)\right)$, the totally bounded uniformity on $A$ which has a base sets of the form

$$
U(\epsilon, K)=\{(s, t)|| f(s)-f(t) \mid<\epsilon \forall f \in K\},
$$

where $\epsilon>0$ and $K$ is a finite subset of $X$, resp. $C^{*}(X)$. (We write $U(\epsilon, f)$ instead of $U(\epsilon,\{f\})$ for simplicity.) The lemma which follows now will be useful in proving Theorem 1 .

Lemma 1. The uniformities $\mathcal{U}(A, X)$ and $\mathcal{U}\left(A, C^{*}(X)\right)$ are identical. Also, the functions that are uniformly continuous with respect to $U(A, X)$ are precisely the members of $C^{*}(X)$.

Proof. Clearly $\mathcal{U}(A, X) \subset U\left(A, C^{*}(X)\right)$. The reverse inclusion is a consequence of the following easily verified statements:

(i) $U(\epsilon, c f)=U(\epsilon /|c|, f)$ for $c \neq 0$.

(ii) $U(\epsilon, f g) \supset U(\epsilon \mid k,\{f, g\})$ whenever $k=\max \{\|f\|,\|g\|\} \times 2 \neq 0$.

(iii) $U(\epsilon, f+g) \supset U(\epsilon / 2,\{f, g\})$.

(iv) $U\left(\epsilon, f_{0}\right) \supset U(\epsilon / 3, f)$ if $\left\|f_{0}-f\right\| \leqq \epsilon / 3$.

(Here $\|f\|$ denotes the usual supremum norm of $f \in C(A)$.)

That every function in $C^{*}(X)$ is uniformly continuous with respect to $U(A, X)$ follows trivially from the first statement of the lemma. Suppose 


$$
f \in C(A), \quad f \notin C^{*}(X),
$$

and $\mathscr{D}$ is the $C^{*}$-subalgebra of $C(A)$ generated by $f$ and $C^{*}(X)$. Then $i^{*}$, the adjoint of the inclusion map $i: C^{*}(X) \rightarrow \mathscr{D}$ is a continuous map of the spectrum $\sigma(\mathscr{D})$ of $\mathscr{D}$ onto the spectrumrof $C^{*}(X)$ and there must exist $x_{1}, x_{2} \in \sigma(\mathscr{D})$ such that $x_{1} \neq x_{2}$ but $i^{*}\left(x_{1}\right)=i^{*}\left(x_{2}\right)$. (Otherwise, $i^{*}$ is a $1-1$ continuous map of one compact Hausdorff space onto another and hence is a homeomorphism, which implies $\mathscr{D}=C^{*}(X)$ and, in particular, $f \in C^{*}(X)$.) It follows that

$$
x_{1}(f)=c_{1} \neq c_{2}=x_{2}(f) .
$$

Also, $\exists$ nets $\left\{s_{\alpha}\right\},\left\{t_{\beta}\right\} \subset A$ such that

$$
g\left(s_{\alpha}\right) \rightarrow x_{1}(g) \text { and } g\left(t_{\beta}\right) \rightarrow x_{2}(g) \forall g \in \mathscr{D} .
$$

And both nets $\left\{g\left(s_{\alpha}\right)\right\}$ and $\left\{g\left(t_{\beta}\right)\right\}$ converge to $\left(i^{*}\left(x_{1}\right)\right) g \forall g \in$ $C^{*}(X)$. Thus, for every $U(\epsilon, K) \in \mathcal{U}(A, X),\left(s_{\alpha}, t_{\beta}\right) \in \mathcal{U}(\epsilon, K) \forall$ large enough $\alpha$ and $\beta$. However $\left|f\left(s_{\alpha}\right)-f\left(t_{\beta}\right)\right| \geqq\left|c_{1}-c_{2}\right| / 2 \forall$ large enough $\alpha$ and $\beta$. Hence $f$ is not uniformly continuous with respect to $U(A, X)$.

Proof of Theorem 1. For $X \subset C(A)$, there is a canonical map $e_{X}$ from $A$ into $C^{*}(X)^{*}$, the dual space of $C^{*}(X) ; e_{X}$ is defined by

$$
\left(e_{X}(s)\right) f=f(s) \forall f \in C^{*}(X) .
$$

If $C^{*}(X)^{*}$ is given the $w^{*}$-topology $\sigma\left(C^{*}(X)^{*}, C^{*}(X)\right)$, then $e_{X}$ is continuous and, if $e_{X}^{*}$ is the adjoint of $e_{X}$, then $e_{X}=\left(e_{X}^{*}\right)^{-1}$ is an isometric isomorphism of $C^{*}(X)$ into $C\left(e_{X}(A)\right)$.

All that has been said above for $A$ and $X \subset C(A)$ applies equally well to $B$ and $Y \subset C(B)$, and we observe that the map $\psi: e_{Y}(s) \rightarrow e_{X}(s)$ effects a uniform isomorphism of $e_{Y}(A)=\left\{e_{Y}(s) \mid s \in A\right\} \subset e_{Y}(B)$ with the relativization to $e_{Y}(A)$ of the uniformity $U\left(e_{Y}(B), e_{Y}(Y)\right)$ onto $e_{X}(A)$ with the uniformity $U\left(e_{X}(A), \mathbf{e}_{X}(X)\right)$; this follows directly from $(*)$ in the statement of Theorem 1 .

So, suppose we are given $f \in C^{*}(X)$. Putting $\hat{f}=e_{X}(f)$, we have from Lemma 1 that $\hat{f}$ is uniformly continuous with respect to $\mathcal{U}\left(e_{X}(A), e_{X}(X)\right)$. Hence $\breve{f}=\psi^{*}(\hat{f}) \in C\left(e_{Y}(A)\right)$ is uniformly continuous with respect to the restriction to $e_{Y}(A)$ of $U\left(e_{Y}(B), e_{Y}(Y)\right)$. If we denote by $\overline{e_{Y}(B)}$ the completion with respect to $U\left(e_{Y}(B), e_{Y}(Y)\right)$, then $e_{Y}(B)$ is compact and the completion $\overline{e_{Y}(A)}$ of $e_{Y}(A)$ with respect to (the restriction to $e_{Y}(A)$ of) $U\left(e_{Y}(B), e_{Y}(Y)\right)$ is a compact subset of $\overline{e_{Y}(B)}$. It then follows from elementary uniform space theory that $\check{f}$ extends to a function $\tilde{f} \subset C\left(\overline{e_{Y}(A)}\right)$ and from the Tietze extension theorem that $\tilde{f}$ has an extension $\bar{f}$ to all of $\overline{e_{Y}(B)}$ satisfying $\|\bar{f}\|=$ 
$\|\tilde{f}\|(=\|\check{f}\|=\|\hat{f}\|=\|f\|)$. Finally, if we apply $e_{Y}^{*}$ to the restriction of $\bar{f}$ to $e_{Y}(B)$, we get the desired function $F$.

A fact that thas not been explicitly used, but is very near the surface in the proof of Theorem 1, is that, for example, the completion of $e_{X}(A)$ with respect to $U\left(e_{X}(A), e_{X}\left(C^{*}(X)\right)\right.$ is just the $w^{*}$-closure in $C^{*}(X)^{*}$ of $e_{X}(A)$, which is the spectrum of $C^{*}(X)$.

Some results of Berglund [1] can be derived as corollaries of Theorem 1. (The first corollary below is a slight improvement of the corresponding result of Berglund.) We need first some notation. $S$ will denote a semitopological semigroup, i.e., a Hausdorff topological space with a separately continuous associative multiplication, and $U(S)$ is defined to be the $C^{*}$-subalgebra of $C(S)$ generated by the coefficients of finite dimensional unitary representations of $S . U(S)$ is called the strongly almost periodic subspace of $C(S)$ and equals the almost periodic subspace $A P(S)$ of $C(S)$ if $S$ is algebraically a group.

Corollary 1 (Berglund [1; Theorem 9]). Suppose that $T$ is a subsemigroup of a semitopological semigroup $S$ and that every coefficient of a continuous finite dimensional unitary representation of $T$ extends to a coefficient of a continuous finite dimensional unitary representation of $S$. Then every member of $U(T)$ extends without increase in norm to a member of $U(S)$.

COROllary 2 (Berglund [1; Corollary 11]). Let $H$ be a subgroup of a locally compact abelian topological group $G$. Then every function almost periodic on $H$ extends to a function almost periodic on $G$.

Proof. The first step of the proof here is the same as in [1]. By Lemma 7 of [1], every continuous character on $H$ extends to a continuous character on $\bar{H}$, which in turn extends to a character on $G$ [5; (24.12) Corollary, p. 380]. Theorem 1 then establishes the corollary once we recall that, for an abelian topological group, the space of almost periodic functions is equal to the $C^{*}$-subalgebra of the continuous functions generated by the continuous characters.

3. Weakly almost periodic functions. In Corollary 2 the hypothesis that $G$ is abelian is crucial. We gave an example in [7] of a locally compact topological group $G$ with closed normal abelian subgroup $H$ such that no nontrivial character on $H$ extends to a function (left and right) uniformly continuous on $G$. Thus such characters do not even extend to functions in $W A P(G)$, the space of functions weakly almost periodic on $G$ [4; Theorem 3.11, p. 42]. (See [2] or [4] for the general theory of weakly almost periodic functions.) However, there 
are settings in which every weakly almost periodic function on a subgroup extends to a function weakly almost periodic on the containing group. A setting where this is obviously the case is now presented. The proof, whose details are omitted, depends on the fact that functions vanishing at infinity on locally compact groups are weakly almost periodic [4; Corollary 3.7 (iii), p. 41].

Proposition 1. Let $H$ be a compact subgroup of a locally compact group $G$. Then every function in $W A P(H)$ extends to a function in $W A P(G)$.

In the setting of Proposition $1 C(H)=W A P(H)=A P(H)$, and one might wonder if the assertion of the proposition is true with $W A P(H)$ and $W A P(G)$ replaced by $A P(H)$ and $A P(G)$, respectively. The following example illustrates that this is not always the case ${ }^{1}$.

EXAMPLE 1. Let $G$ be the group of finite permutations of the natural numbers; each member of $G$ permutes only a finite number of natural numbers. It has been communicated to us by W. A. Veech (and follows from results in [3; Ch. IV]) that the only functions in $A P(G)$ are linear combinations of the constant function 1 and the function that is 1 at even permutations and -1 at odd permutations. It follows easily from this fact that, if $H$ is any finite subgroup of $G$ containing more than 3 elements, then not all functions in $A P(H)$ extend to functions in $A P(G)$.

It is known that weakly almost periodic functions on a subgroup $H$ of a topological group $G$ extend to functions in $W A P(G)$ in the following settings:

(i) $\quad H$ is dense in $G$ [7; Theorem 4.9 (i)].

(ii) $G$ is locally compact and abelian and $H$ is closed [4; Theorem 3.16 , p. 49].

(iii) $G$ is locally compact and $H$ is normal and open [4; Theorem 3.14, p. 47].

By using (i), one can see that the requirement that $H$ be closed is unnecessary in (ii). A similar comment can also be made about (iii) and about Theorem 2 here, which is a generalization of (iii).

THEOREM 2. Let $H$ be an open subgroup of a locally compact group $G$. Let $\psi$ be the map that extends functions $f$ on $H$ to functions $F$ on $G$ by the formula

' The referee remarks that the failure of almost periodic functions on a compact subgroup of a locally compact group to extend as such to the whole group is well known via the example of the special unitary group $S U(2)$ in the special linear group $S L(2)$. 


$$
\psi f(s)=F(s)=\left\{\begin{array}{l}
f(s) \text { if } s \in H \\
0 \text { otherwise. }
\end{array}\right.
$$

Then $\psi$ is an isometric injection of $W A P(H)$ into $W A P(G)$.

Proof. The proof is much the same as, but, if anything, a little simpler than, that of (iii) in [4]. The problem is to show that, if $f \in W A P(H)$, then $F=\psi f \in W A P(G)$, i.e., to show that any sequence $\left\{F_{s_{n}}\right\}$ of left translates of $F$ has a weakly convergent subsequence. (Here the left translate $F_{s}$ of $F \in C(G)$ by $s \in G$ is defined by $F_{s}(t)=F(s t) \forall t \in G$.) We begin by fixing coset decompositions of $G$,

$$
G=U H t_{\beta}=U t_{\beta}^{-1} H
$$

Without loss we may assume either

(a) $\exists t_{\beta_{0}}=t_{0}$ such that $\left\{s_{n}\right\} \subset H t_{0}$, or

(b) The statements $n \neq m, s_{n} \in H t_{\beta_{n}}, s_{m} \in H t_{\beta_{m}}$ imply that $t_{\beta_{n}} \neq t_{\beta_{m}}$.

In case (a), $s_{n}=r_{n} t_{0}$ for some $r_{n} \in H$ and we may assume that $\left\{f_{r_{n}}\right\}$ converges weakly in $C(H)$ to $d \in C(H)$. It follows that $\left\{F_{r_{n}}\right\}$ converges weakly in $C(G)$ to $D=\psi d \in C(G)$ and hence that $\left\{F_{s_{n}}\right\}=\left\{\left(F_{r_{n}}\right)_{t_{0}}\right\}$ converges weakly in $C(G)$ to $D_{t 0}$. (Here we are using the fact that left translation is a weak homeomorphism of $C(G)$.)

In case (b), $s_{n}=r_{n} t_{\beta_{n}}$ for some $r_{n} \in H$ and some $t_{\beta_{n}}, t_{\beta_{n}} \neq t_{\beta_{m}}$ if $s_{n} \neq s_{m}$, and $F_{s_{n}}(t)=0$ if $s_{n} t \notin H$, i.e., if $t \notin s_{n}^{-1} H=t_{\beta_{n}}^{-1} H$. Thus the functions $\left\{F_{s_{n}}\right\}$ have disjoint supports. Suppose $\mu \in C(G)^{*}$. We will show $\mu\left(F_{s_{n}}\right) \rightarrow 0$. Suppose, to the contrary, that $x \neq 0$ is a cluster point of the sequence $\left\{\mu\left(F_{s_{n}}\right)\right\}$ and suppose $\left\{\mu\left(F_{s_{n}}\right)\right\}$ is a subsequence such that $\left|\mu\left(F_{s_{n i}}\right)-x\right| \leqq|x| / 2 \forall i$. Then

$$
\begin{gathered}
F_{m}=\sum_{i=1}^{m} F_{s_{n i}} \in C(G) \forall m=1,2,3, \cdots,\left\|F_{m}\right\|=\|F\| \text {, and } \\
\left|\mu\left(F_{m}\right)\right|=\left|\sum_{i=1}^{m} \mu\left(F_{s_{n i}}\right)\right| \geqq m|x| / 2,
\end{gathered}
$$

which is greater than $\|\mu\|\|F\|$ for large enough $m$. This is a contradiction and we are done.

From Theorem 2 it follows that any function weakly almost periodic on any subgroup of the group $G$ of Example 1 extends to a function weakly almost periodic on $G$. (We remark that the only normal subgroup of $G$ is the group of even permutations; this is a consequence of remarks in [3; Ch. IV].) 
Added in proof. Dr. H. D. Junghenn has informed us that Theorem 1 can be quickly proved using a general theorem about $C^{*}$-algebras. (See 1.8.3 Corollaire of J. Dixmier's Les $C^{*}$-algèbres et leurs représentations, Gauthier-Villars, Paris, 1964).

\section{REFERENCES}

1. J. F. Berglund, On extending almost periodic functions, Pacific J. Math., 33 (1970), 281-289.

2. J. F. Berglund and K. H. Hofmann, Compact Semitopological Semigroups and Weakly Almost Periodic Functions, Lecture Notes in Mathematics, 42, Springer-Verlag, Berlin, 1967.

3. H. Boerner, Representations of Groups, North-Holland, Amsterdam, 1963.

4. R. B. Burckel, Weakly Almost Periodic Functions on Semigroups, Gordon and Breach, New York, 1970.

5. E. Hewitt and K. Ross, Abstract Harmonic Analysis I, Springer-Verlag, Berlin, 1963.

6. J. L. Kelley, General Topology, D. Van Nostrand, Princeton, 1955.

7. P. Milnes, Compactifications of semitopological semigroups, J. Australian Math. Soc., 15 (1973), 488-503.

Received November 26, 1973 and in revised form February 1, 1974. This research was supported in part by NRC grant A7857.

University of Western Ontario, Canada 




\section{Pacific Journal of Mathematics}

\section{Vol. 56, No. $1 \quad$ November, 1975}

Shimshon A. Amitsur, Central embeddings in semi-simple rings .......... 1

David Marion Arnold and Charles Estep Murley, Abelian groups, A, such

that $\operatorname{Hom}(A,---)$ preserves direct sums of copies of $A \ldots \ldots \ldots .$.

Martin Bartelt, An integral representation for strictly continuous linear

operators ................................... 21

Richard G. Burton, Fractional elements in multiplicative lattices......... 35

James Alan Cochran, Growth estimates for the singular values of

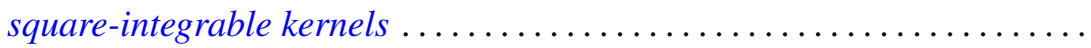

C. Martin Edwards and Peter John Stacey, On group algebras of central

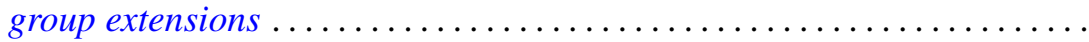

Peter Fletcher and Pei Liu, Topologies compatible with homeomorphism

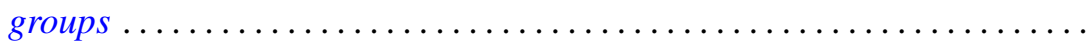

George Gasper, Jr., Products of terminating ${ }_{3} F_{2}(1)$ series ............ 87

Leon Gerber, The orthocentric simplex as an extreme simplex ............

Burrell Washington Helton, A product integral solution of a Riccati

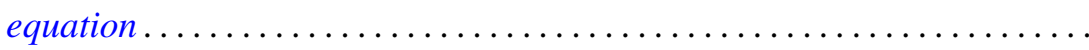

Melvyn W. Jeter, On the extremal elements of the convex cone of

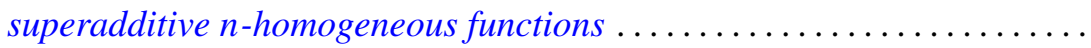

R. H. Johnson, Simple separable graphs .

Margaret Humm Kleinfeld, More on a generalization of commutative and

alternative rings. . .

A. Y. W. Lau, The boundary of a semilattice on an $n$-cell.

Robert F. Lax, The local rigidity of the moduli scheme for curves ...

Glenn Richard Luecke, A note on quasidiagonal and quasitriangular

operators .

Paul Milnes, On the extension of continuous and almost periodic functions

Hidegoro Nakano and Kazumi Nakano, Connector theory.

James Michael Osterburg, Completely outer Galois theory of perfect rings ..................................

Lavon Barry Page, Compact Hankel operators and the F. and M. Riesz

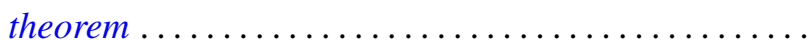

Joseph E. Quinn, Intermediate Riesz spaces..................... 225

Shlomo Vinner, Model-completeness in a first order language with a generalized quantifier.

Jorge Viola-Prioli, On absolutely torsion-free rings ..........

Philip William Walker, A note on differential equations with all solutions of integrable-square............................

Stephen Jeffrey Willson, Equivariant maps between representation 